\title{
Chemistry of labile small sulfur allotropes in interactive coordination networks \\ Hiroyoshi Ohtsu $^{1}$ \\ ${ }^{1}$ Department Of Chemistry, Tokyo Institute Of Technology, Meguro-ku, Japan \\ E-mail: ohtsu@chem.titech.ac.jp
}

Sulfur has more than 30 kinds of allotropes such as stable octasulfur S8, metastable S6, S12, polymer chain and so on. However, isolation and characterization of small sulfur allotropes $(\mathrm{Sn}, \mathrm{n}<6)$ have long remained unachievable because of their extreme lability. In this study, we report the first direct X-ray observation of small sulfur allotropes and their reactions in interactive pores of porous coordination networks.

We isolated one of the reactive small sulfur, bent-S3 (trisulfur), in crystalline powder of porous coordination network, [(ZnI2)3(TPT)2] ( TPT = 2,4,6-tris(4-pyridyl)-1,3,5-triazine ). The sulfur encapsulation was performed under thermodynamic conditions using sulfur vapor: the network powder was heated with sulfur (S8) at $533 \mathrm{~K}$ for 6 hours under vacuum. The structure of sulfur-encapsulating network was determined by ab initio XRPD (X-ray powder diffraction) analysis: we observed disordered bent-S3 in a pore of the network. The S3 in a pore is stable up to $500 \mathrm{~K}$ thanks to the stabilization by interaction of sulfur with interactive pore site. Furthermore, the S3 in a pore can convert to S6 by grinding the powder or heating with ammonium chloride, showing flexibility of the network.

To clarify the process of the small sulfur encapsulation, we performed kinetic trapping of small sulfur into single crystals of two interactive network isomers, [(CuI)2(TPPM)] (TPPM = tetra-4-(4-pyridyl)phenylmethane ). Both networks encapsulate disulfur, S2, kinetically. One of the networks displayed shape selectivity for linear molecules only, therefore S2 was trapped and remained stable within the network at room temperature and higher. In the second network, however, the S2 molecules reacted further to produce bent-S3 species as the temperature was increased. Following the thermal evolution of the $\mathrm{S} 2$ species in this network with X-ray diffraction and Raman spectroscopy unveiled the generation of a new reaction intermediate never observed before, cyclo-trisulfur dication (cyclo-S3(2+)). We clarified the conversion reaction of small sulfur, S2 to bent-S3 via cyclo-S3(2+), directly by X-ray. We envisage kinetic guest trapping in interactive crystalline porous networks as a promising method to investigate transient chemical species.

In this study, we revealed the crystal structure of labile sulfur allotropes: S2, bent-S3 and cyclo-S3. We observed small sulfur conversion reaction in a pore by direct X-ray observations. This study shows new chemistry of sulfur allotropes.

[1] Ohtsu, H. et al. (2013) J. Am. Chem. Soc. 135, 11449-11452.

[2] Kitagawa, H. et al. (2016) IUCr] 3, 232-236.

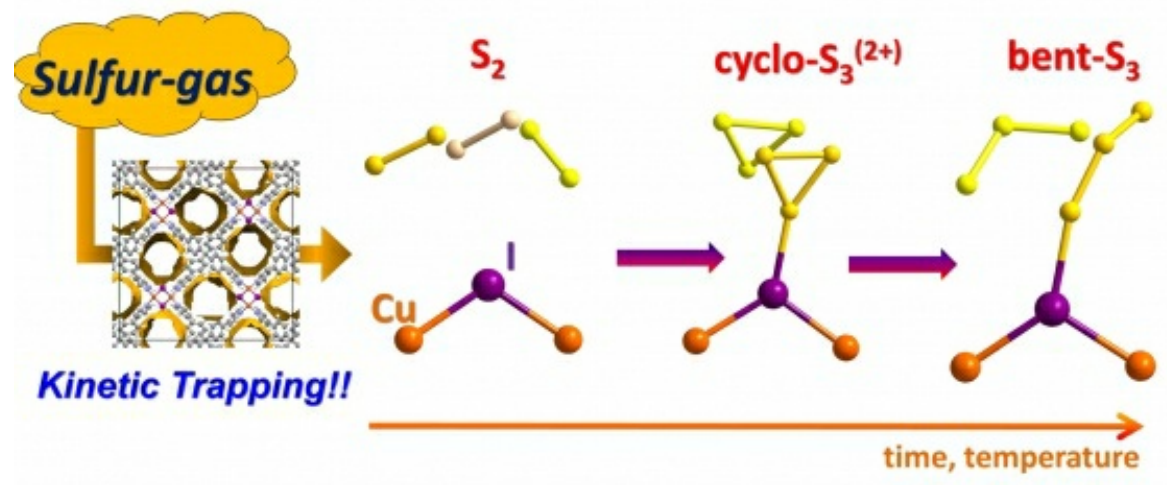

Direct observation by $X$-ray diffraction

Keywords: Small sulfur allotropes, Reaction intermediates, Porous coordination networks 